\section{Spontaneous Coronary Artery Dissection in a Middle-aged Woman with Acute Anterior Myocardial Infarction}

Sir,

Spontaneous coronary artery dissection (SCAD) is a highly unusual cause of acute coronary disease. ${ }^{1}$ It is the result of a hematoma formation within the outer third of the tunica media, with subsequent expansion leading to compression of the true lumen and resultant myocardial ischemia. The most common presentation is acute coronary syndrome, but the severity may range from unstable angina to acute myocardial infarction and cardiogenic shock. This disease is associated with a low clinical detection rate and a series of ranges from $0.1 \%$ to $0.28 \%$. Many patients are misdiagnosed or never diagnosed. As a result, SCAD has a high mortality rate, if not identified and treated promptly. ${ }^{2}$

SCAD has been observed in patients with coronary disease, and in women in their perinatal period or with the use of oral contraceptives. There is a predominance of SCAD in women $(75 \%)$. It is related to the elevation of estrogen and progesterone. Hormonal changes weaken the coronary artery walls and contribute to degeneration and dissection. LAD involvement is more common in females and right coronary artery (RCA) involvement is more common in males. ${ }^{3} \mathrm{~A}$ middle-aged woman who had been taking contraceptives had irregular menstruation with menopausal indication in the recent two years. There was no past history of cardiovascular disease and relevant positive family history. There was a sudden onset of chest pain with intense physical activity. The ECG was abnormal (Figures 1a and 1b), and coronary angiography revealed lesions of LAD progressing rapidly (Figures $1 \mathrm{c}$ and $1 \mathrm{~d}$ ). The first angiography failed to detect the coronary artery dissection. Subsequent further expansion of the dissection and total occlusion of LAD led to the formation of a left ventricular aneurysm (Figure 1e).

The diagnosis is usually confirmed by coronary angiography, but it has some limitations including the precise localisation of the dissection entry point and the identification of the true and false lumen. ${ }^{4}$ This may require intravascular imaging modalities such as intravascular ultrasound (IVUS). IVUS can display the vascular cross-sectional images clearly and analyse quantitatively, providing a complementary role in the diagnosis of SCAD.

SCAD is a rare but serious cause of acute coronary syndrome. It should be suspected in young to middleaged women presenting with chest pain, even in the absence of cardiovascular risk factors. One should be able to obtain a detailed past history and analyse coronary angiography results carefully. If necessary, IVUS may help in further examinations to reduce the incidence of erroneous diagnosis or missed diagnosis. ${ }^{5}$

\section{REFERENCES}

1. Vavuranakis M, Latsios G, Tousoulis D, Vaina S, Triantafyllou G, Drakopoulou $\mathrm{M}$, et al. Spontaneous coronary dissection as a cause of acute coronary syndrome: Evidence for noninflammatory underlying mechanisms. Int J Cardiol 2007; 114: e24-26.

2. Moles VP, Chappuis F, Simonet F, Urban P, De La Serna F, Pande AK, et al. Aortic dissection as complication of percutaneous transluminal coronary. Cathet Cardiovasc Diagn 1992; 26: 8-11.

3. Vanzetto G, Berger-Coz E, Barone-Rochette G, Chavanon O, Bouvaist $\mathrm{H}$, Hacini $\mathrm{R}$, et al. Prevalence, therapeutic management and medium-term prognosis of spontaneous coronary artery dissection: Result from a database of 11,605 patients. Eur J Cardiothorac Surg 2009; 35:250-4.

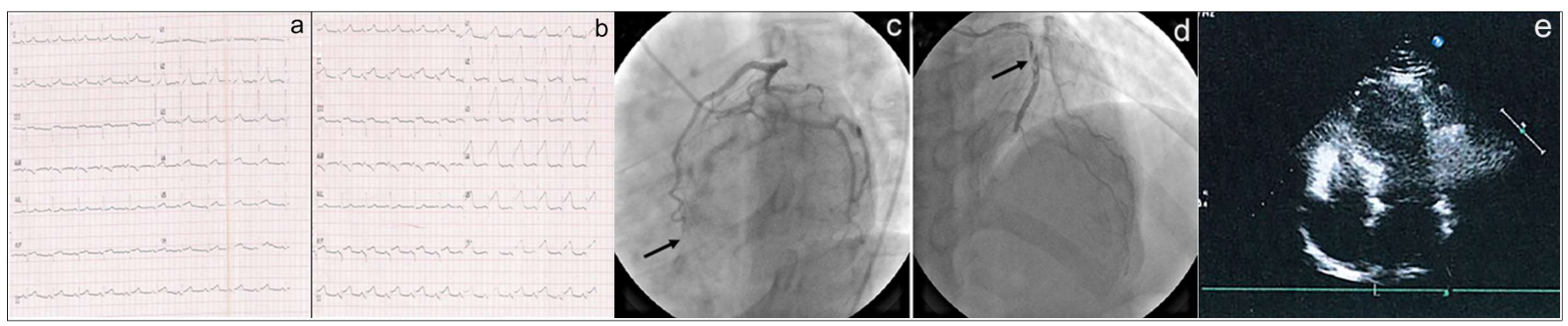

Figure 1: (a) Admission electrocardiogram revealed arched ST segment elevation of $0.2 \mathrm{mv}$ in leads II, III aVF and 0.05-0.2mv in leads V5 and V6. (b) Electrocardiogram during the patient's recurrence of chest pain showed arched ST segment elevation of $0.3 \mathrm{mv}$ in leads II, III aVF; ST segment showed arched elevation of $0.05-0.2 \mathrm{mv}$ and T-wave appeared sharp and high in leads V1-V6. (c) Emergency coronary angiography revealed irregular vascular wall and plaque from the ostium to the first diagonal proximal part of the LAD, which also demonstrated a $50 \%$ localized stenosis of the LAD at its ostium with TIMI grade 3 flow (As the arrow can be seen the dissection which we diagnosed ). (d) Second coronary angiography showed total occlusion of the middle LAD with TIMI Grade 0 flow (As the arrow can be seen the spiral shaped dissection of the proximal and middle LAD). (e) Echocardiogram revealed left ventricular hypertrophy, segmental wall motion abnormalities(interventricular septum, lateral wall, inferior and posterior wall motion disappearance; anterior wall motion abatement), a left ventricular aneurysm was present (occupied $30 \%$ left ventricular area) with left ventricular ejection fraction $45 \%$. 
4. Mortensen $\mathrm{KH}$, Thuesen L, Kristensen IB, Christiansen EH. Spontaneous coronary artery dissection: A Western Denmark heart registry study. Catheter Cardiovasc Interv 2009; 74: 710-7.

5. Kawamori $H$, Shite J, Shinke T, Otake H, Matsumoto D, Nakagawa $\mathrm{M}$, et al. Natural consequence of post-intervention stent malapposition, thrombus, tissue prolapse, and dissection assessed by optical coherence tomography at midterm follow-up. Eur Heart J Cardiovasc Imaging 2013; 14: 865-75.

Xue-Qing Yangl, Hai-Yan Zhu', Xian Wang', Huai-Bing Zhao', Wei Zhang', Min Xiao ${ }^{2}$ and Li-Jing Zhang ${ }^{2}$
1 Department of Cardiovascular, Beijing Hospital of Traditional Chinese Medicine ShunYi Branch, Beijing, 101300, China

2 Department of Cardiovascular, Dongzhimen Hospital Affiliated to Beijing University of Chinese Medicine, Beijing 100700, China

Correspondence: Dr. Li-Jing Zhang, Cardiovascular Department, Dongzhimen Hospital Affiliated to Beijing University of Chinese Medicine, Beijing University of Chinese Medicine Institute for Cardiovascular Disease, No. 5 of HaiYuncang Street, Dongcheng District, Beijing 100700, China E-mail: zhanglj1523@163.com

Received: January 05, 2018; Accepted: September 09, 2018 ......... 